\title{
REPRODUCIBILITY OF MEASUREMENTS OF PRE-CONTACT LENS TEAR FILM KINETICS UNDER NORMAL AND ADVERSE ENVIRONMENTAL CONDITIONS
} Michel Guillon ${ }^{1}$, PhD, FCOptom, FAAO, FBCLA, CCTI, Kishan Patel ${ }^{1}$, Ruchi Gupta ${ }^{1}$, Trisha Patel ${ }^{1}$, Cecile A. Maissa ${ }^{2}$

${ }^{1}$ OCULAR TECHNOLOGY GROUP - International

${ }^{2}$ Alcon Research, LLC

Corresponding author: mguillon@otg.co.uk

Submitted: 12 February, 2021. Accepted: 27 August, 2021. Published: 30 September, 2021.

\section{ABSTRACT}

Objectives: To evaluate the reproducibility of tear film kinetics (TFK) measurements during the full interblink period in habitual wearers of delefilcon A daily disposable contact lenses under different environmental conditions.

Methods: Two independent studies were performed, one involving 90 eyes measurements in 49 subjects and the second involving 58 eyes measurements in 32 subjects, after 3 hours of conventional wear in normal environmental conditions (NEC) and an additional 3 hours of computer use under adverse environmental conditions (AEC), defined as $20 \%$ relative humidity (RH). Digital videos were obtained by non-invasive Tearscope illumination, followed by a parallel-group post-hoc comparative analysis by masked investigators of the videos obtained during the two studies. Parameters analyzed included non-invasive break-up time (NIBUT); dehydration speed (DS) between the initial break and the spontaneous blink, and minimum protected area (MPA) of the lens surface by the tear film.

Results: After 3 hours of wear in normal environmental conditions, the overall population $(n=148)$ had a mean NIBUT of $7.1 \pm 7.0 \mathrm{sec}$, a mean DS of $0.28 \pm 0.66 \mathrm{~mm}^{2} / \mathrm{sec}$, and a mean MPA of $93.4 \pm 16.6 \%$. After an additional 3 hours in AEC, mean NIBUT was $8.4 \pm 9.8 \mathrm{~mm}^{2} / \mathrm{sec}$, mean DS was $0.26 \pm 0.75 \mathrm{~mm} / \mathrm{sec}$, and MPA was $95.2 \pm 14.0 \%$. The TFK characteristics in the two studies were similar for each environmental condition: after 3 hours of conventional wear, the mean $[95 \% \mathrm{CI}]$ differences between the two studies were $-0.9[-3.2,+1.4] \mathrm{sec}$ for NIBUT, $0.00[-0.22,+0.22] \mathrm{mm}^{2} / \mathrm{s}$ for DS, and $2.0[-3.5,+7.4] \%$ for MPA; after an additional 3 hours of wear in $20 \% \mathrm{RH}$, the mean $[95 \% \mathrm{CI}]$ differences between the two studies were $-1.3[-4.5,+1.9] \mathrm{sec}$ for NIBUT, $-0.03[-0.28,+0.22] \mathrm{mm}^{2} / \mathrm{s}$ for DS and $-0.3[-4.9,+4.3] \%$ for MPA.

Conclusions: This analysis showed that the measurement of pre-contact lens TFK carried out in two different studies involving habitual wearers of delefilcon A having worn their contact lenses for an initial 3 hours under NEC and a further 3 hours under low (20\%) hygrometry (AEC) produced similar on-eye wettability,

J Cont Lens Res Sci Vol 5(1):e11-e30; September 30, 2021

This article is distributed under the terms of the Creative Commons Attribution-

Non Commercial 4.0 International License. (CGuillon et al. 
including a long NIBUT, slow DS following the initial break, and wide MPA at the next blink. The results establish the reproducibility of the measurement of pre-contact lens TFK using the Tearscope; by quantifying the measurement variability, the data makes it possible to carry out precise sample size calculations in future studies involving pre-contact lens TFK measurements to quantify on-eye wettability under both normal and adverse environmental low (20\% RH) hygrometric conditions.

Keywords: Tear film kinetics. repeatability, reproducibility, delefilcon A, adverse environmental conditions (AEC)

\section{INTRODUCTION}

Tear film break-up time, or the interval between a complete blink and the appearance of the first break in the tear film, is considered a clinical marker of tear film stability. ${ }^{1}$ Various non-invasive techniques have been used to measure tear film break-up time in contact lens wearers, including interferometry, the Tearscope, and videokeratoscopy. ${ }^{1}$ The drawback of limiting the assessment of tear film stability during the interblink period when measuring non-invasive break-up time (NIBUT) is that it only provides information of wettability on that period. These assessments cannot determine the nature and severity of the break from that time until the next spontaneous blink. Knowing the status of the contact lens front surface at the time of the blink is essential, as it determines the influence of the friction of the eyelid that occurs during the blink, a factor associated with contact lens comfort. ${ }^{2,3}$ The advent of high-resolution video recording has led to capturing the pre-contact lens tear film illuminated by the Tearscope over the full interblink period and quantifying the tear film characteristics over the whole period via post hoc analysis. ${ }^{3}$ A similar approach has been implemented via the analysis of distortion of videokeratoscope concentric rings to quantify the tear film changes during the interblink period. ${ }^{1}$

A key characteristic of any measurement technique is its validity. TFOS DEWS II Tear Film sub-committee

acknowledged this to be an issue when measuring tear film stability. ${ }^{4}$ In summarizing issues regarding the measurement of the tear film break-up time with fluorescein the committee stated that it has an "inherent variability." Even when considering the non-invasive measurement of the tear film break-up time, it stated that the techniques had "reasonable agreement that they operate with a coefficient of variation of around 10\%".
The committee quoted the "coefficient of variation" as an indicator of NIBUT measurement validity. The coefficient of variation is obtained by dividing the population standard deviation by the population mean, which indicates the between-people variability within the population but not the validity of the measurement. Basic clinical metrology principles identify repeatability and reproducibility as the key measurements validating parameters. ${ }^{5}$ Repeatability refers to the variation in repeated measurements obtained over a short time under identical conditions, while reproducibility refers to measurements made under different conditions, such as different operators, conducted over an extended time. Finally, repeatability is often reported as the standard deviation of the difference between the two sets of measurements in clinical studies. This approach overestimates by approximately a factor of two repeatability, as repeatability standard reporting in metrology is $95 \%$ confidence interval.

To test the reproducibility of the method, we needed a stable system. We, therefore, selected delefilcon A (DAILIES TOTAL1, Alcon Laboratories, Fort Worth, TX) silicone hydrogel contact lenses, which have highly repeatable on-eye performance. Delefilcon $\mathrm{A}$ is an advanced generation silicone hydrogel polymer formulated with phosphatidylcholine, ${ }^{6,7}$ a component of natural tears that plays a key role in the maintenance of the tear film. ${ }^{8}$ These lenses, first made commercially available in 2013 , contain $33 \%$ water distributed in a core-to-surface water gradient over a 1-2 micron transition zone ranging from $33 \%$ at the core to $\sim 80 \%$ at the front and back surfaces. ${ }^{6,7}$

The objective of this study was to evaluate the reproducibility of tear film kinetics (TFK) measurements during the full interblink period in habitual wearers

J Cont Lens Res Sci Vol 5(1):e11-e18; September 30, 2021

This article is distributed under the terms of the Creative Commons Attribution-

Non Commercial 4.0 International License. (C) Guillon et al. 
of delefilcon A daily disposable contact lenses under different environmental conditions.

\section{METHODS}

\section{Study population}

The intention to treat the population of this study consisted of habitual wearers of delefilcon A soft contact lenses. Study 1 involved 90 eyes with valid measurements in 49 subjects, and Study 2 involved 58 eyes with valid measurements in 32 subjects. Thus, a total of 81 subjects were included in this study. All subjects were aged $\geq 18$ years and had worn delefilcon A contact lenses for at least 2 months. Subjects were required to have vision correctable to $6 / 7.5$ or $0.1 \log$ MAR or better in each eye at a distance with the study contact lenses at the time of screening and to be willing to wear their contact lenses every day or at least for a minimum of 5 days per week for 6 hours per day during the study period. Participants had to discontinue artificial tears and rewetting drops on the days of study visits. Subjects with any ocular anterior segment infection, inflammation, abnormality, or disease were excluded, or who used any systemic or ocular medications for which contact lens wear could be contraindicated. Subjects could not have participated in any other clinical study within 30 days before enrollment. The Ethics Committees approved the protocols of the two separate studies under the UK IRAS centralized ethics committee system, and all subjects provided written informed consent prior to testing.

\section{Study design}

The current analysis is a post-hoc parallel-group comparison of the measurements of pre-contact lens TFK obtained in two separate clinical studies, carried out approximately one year apart, following the same protocol and measurement conditions to determine measurement reproducibility.

The clinical protocol for the two studies required each subject to have worn delefilcon A contact lenses for $10 \pm 3$ days before the day of the study visit. After each wearing period, contact lens wettability was assessed twice using the Tearscope (Keeler, Windsor, UK), once after three hours of wear under normal environmental conditions (NEC) and again after a further three hours of wear while using a computer in a setting of $20 \%$ relative humidity $(\mathrm{RH})$, representing an AEC or dry environment typical of an air-conditioned office.

\section{Maintenance of $20 \% \mathrm{RH}$}

The $20 \%$ RH was maintained using an environmental ocular goggle device designed and built by OCULAR TECHNOLOGY GROUP-International using modified welding googles and a portable instrument that can maintain relative humidity between $0 \%$ and $80 \%$. The instrument comprises a portable attaché case containing the battery-operated system that produces constant airflow at the required speed and humidity and a pair of goggles that creates the constant controlled environment around the eyes.

\section{Tearscope analysis}

TFK analysis was based on digital videos of the tear film over the total interblink period for each subject. The tear film was visualized using the Tearscope diffuse lighting system attached to a Topcon DC4 slit lamp (Topcon, Newbury, UK) set at x25 magnification; recordings were captured with the Topcon DV3 digital camera. ${ }^{1}$ The video recordings were masked and randomized before analysis by trained personnel. The parameters analyzed included NIBUT, defined as the time elapsed (seconds) between eye opening after a blink and the appearance of the first dark spot (break in the tear film) within the tear film (Figure 1A); minimum protected area (MPA), defined as the percent $(\%)$ of the lens surface covered by the tear film that occurs immediately before the next blink (Figure 1B); and dehydration speed (DS), defined as the speed of increase of uncovered area of the lens after the first break in the tear film (i.e., the area in Figure 1B minus the area in Figure 1A) divided by time and reported as $\mathrm{mm}^{2} / \mathrm{sec}$. NIBUT was measured for three successive interblink periods, and the TFK analyzed for the interblink period corresponding to the median NIBUT.

\section{Statistical analyses}

Data were analyzed using SAS 9.2 statistical software (SAS Institute, Cary, NC). Two data points were recorded; after an initial 3 hours of wear under normal environmental conditions and after a further

J Cont Lens Res Sci Vol 5(1):e11-e18; September 30, 2021

This article is distributed under the terms of the Creative Commons Attribution-

Non Commercial 4.0 International License. (C) Guillon et al. 
A)

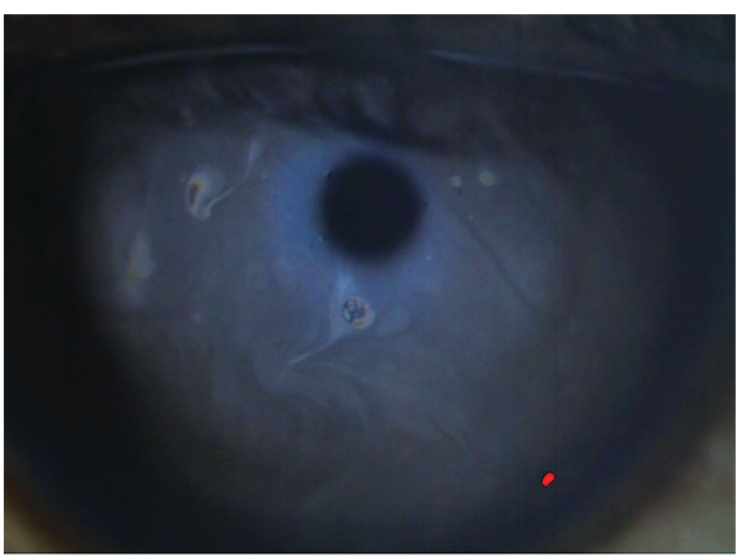

B)

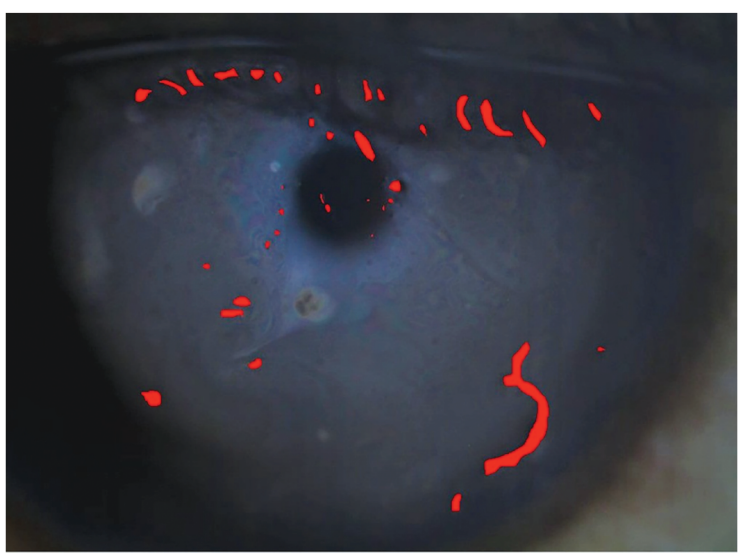

Figure 1. Examples of exposed areas, as assessed by the Tearscope, (A) at first break and (B) immediately before the blink.

3 hours of wear and exposure to 20\% RH. Descriptive statistics for categorical data are reported as the number of subjects (n) and the frequency (\%), and for continuous data as mean, standard deviation (SD), median, minimum, maximum, and $95 \%$ confidence interval (CI). Measurement reproducibility, as per accepted metrology standards, is reported by the $95 \%$ confidence level of the mean difference.

\section{RESULTS}

\section{Subject disposition}

The study population consisted of 81 wearers of delefilcon A lenses from two studies, 49 from Study 1 and 32 from Study 2. The study's overall population demographic characteristics are shown in Table 1, and baseline ocular characteristics are shown in Table 2.

Overall the subjects included 35\% men (33\% Study 1, 38\% Study 2) and 65\% women (67\% Study $1,63 \%$ Study 2), and the mean age was $34.8 \pm 11.8$ years (35.1 \pm 13.1 Study 1 and 34.3 \pm 9.5 Study 2$)$, range 19 to 74 years. Baseline refractive errors were $-3.76 \pm$ $1.87 \mathrm{D}$ spherical $(-3.55 \pm 1.88$ Study $1,-4.07 \pm 1.85$ Study 2$)$, cylinder $-0.36 \pm 0.26 \mathrm{DC}(-0.38 \pm 0.26$ Study $1,-0.33 \pm 0.27$ Study 2$)$ and best corrected decimal Snellen visual acuity $1.23 \pm 0.21(1.23 \pm 0.21$ Study $1,1.23 \pm 0.22$ Study 2$)$. There were no significant differences in mean baseline characteristics between the habitual wearers of delefilcon A contact lenses in the two studies $(\mathrm{p}>0.05)$.

\section{TFK characteristics in normal vs. adverse environments: Studies 1 and 2 Combined NIBUT}

After wearing their habitual delefilcon A lenses for $10 \pm 3$ days, the mean \pm SD NIBUT after the initial 3 hours of conventional wear on the day of the visit was $7.1 \pm 7.0$ seconds. Following exposure to $20 \%$ RH for an additional 3 hours (total, 6 hours of wear), the mean \pm SD NIBUT was $8.4 \pm 9.8$ seconds (Figure 2). Thus, the mean $[95 \% \mathrm{CI}]$ difference for

Table 1. Demographic Characteristics of Wearers of Delefilcon A Lenses Intention-To-Treat Population

\begin{tabular}{|l|c|c|c|}
\hline & Study 1 & Study 2 & Total \\
\hline Number & 49 & 32 & 81 \\
\hline Age, $\mathbf{y r}$ & & & $34.8 \pm 11.84$ \\
\hline Mean \pm SD & $35.1 \pm 13.14$ & $34.3 \pm 9.49$ & 32.0 \\
\hline Median & 32.0 & 32.0 & $19-74$ \\
\hline Range & $19-74$ & $19-56$ & \\
\hline Sex, $\mathbf{n}$ (\%) & & & $28(34.6)$ \\
\hline Male & $16(32.7)$ & $12(37.5)$ & $53(65.4)$ \\
\hline Female & $33(67.3)$ & $20(62.5)$ & \\
\hline
\end{tabular}

J Cont Lens Res Sci Vol 5(1):e11-e18; September 30, 2021

This article is distributed under the terms of the Creative Commons Attribution-

Non Commercial 4.0 International License. (C) Guillon et al. 
Table 2. Baseline Ocular Characteristics of Wearers of Delefilcon A Lenses Intention-To-Treat Population

\begin{tabular}{|l|c|c|c|}
\hline & Study 1 & Study 2 & Total \\
\hline Sphere (D) & & & 162 \\
\hline Number of eyes & 98 & 64 & $-3.76 \pm 1.87$ \\
\hline Mean \pm SD & $-3.55 \pm 1.88$ & $-4.07 \pm 1.85$ & $-3.5(-9.25$ to -0.25$)$ \\
\hline Median (range) & $-3.25(-9.25$ to -0.25$)$ & $-3.75(-8.50$ to -1.00$)$ & 162 \\
\hline Cylinder (D) & & & $-0.36 \pm 0.26$ \\
\hline Number of eyes & 98 & 64 & $-0.32(-0.75$ to 0.00$)$ \\
\hline Mean \pm SD & $-0.38 \pm 0.26$ & $-0.33 \pm 0.27$ & 162 \\
\hline Median (range) & $-0.38(-0.75$ to 0.00$)$ & $-0.25(-0.75$ to 0.00$)$ & $1.23 \pm 0.21$ \\
\hline BCVA (Decimal) (OD \& OS) & & & $1.25(1.0$ to 1.6$)$ \\
\hline Number of eyes & 98 & 64 & $1.23 \pm 0.22$ \\
\hline Mean \pm SD & $1.23 \pm 0.21$ & $1.25(0.9$ to 1.6$)$ & \\
\hline Median (range) & $1.25(1.0$ to 1.6$)$ &
\end{tabular}

NIBUT between the two environments was $-1.3[-4.5$, +1.9 ] seconds.

\section{Dehydration speed (DS)}

Mean \pm SD DS following the initial 3 hours of conventional delefilcon A lens wear was $0.28 \pm 0.66$ $\mathrm{mm}^{2} /$ seconds. After exposure to $20 \% \mathrm{RH}$ for an additional 3 hours (6 hours of wear), the mean \pm SD DS was $0.26 \pm 0.75 \mathrm{~mm}^{2} /$ seconds (Figure 3). The mean $[95 \% \mathrm{CI}]$ difference for DS between the two environments was $-0.03[-0.28,0.22] \mathrm{mm}^{2} /$ seconds.

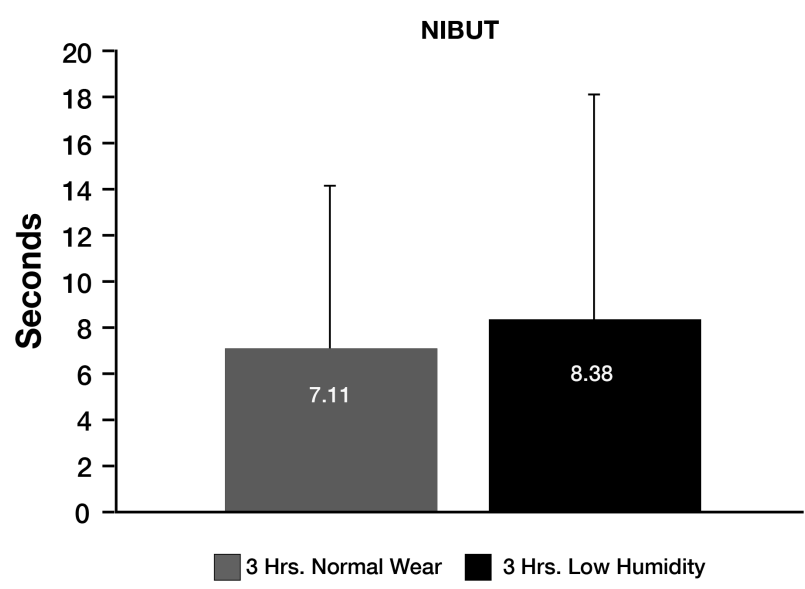

Figure 2. Mean $\pm \mathrm{SD}$ non-invasive break-up time (NIBUT) for habitual delefilcon A wearers after 3 hours of normal wear and 3 hours of exposure to $20 \%$ relative humidity.

\section{MInimum protected area (MPA)}

After wearing their habitual delefilcon A lenses for the initial 3 hours, the mean \pm SD \% MPA was $93.4 \pm 16.6 \%$; after 3 hours at $20 \%$ RH (total, 6 hours of wear), the mean \pm SD \% MPA was $95.2 \pm 14.0 \%$ (Figure 4). The mean [95\% CI] difference for MPA between the two environments was -0.32 [ -4.94 to $4.31] \%$.

TFK characteristics after the initial 3 hours of delefilcon A lens wear were indicative of good on-eye wettability, as evidenced by long pre-contact lens NIBUT, slow dehydration after the initial break, and very high tear film coverage at the time of spontaneous blink. Further, TFK characteristics were unchanged after an

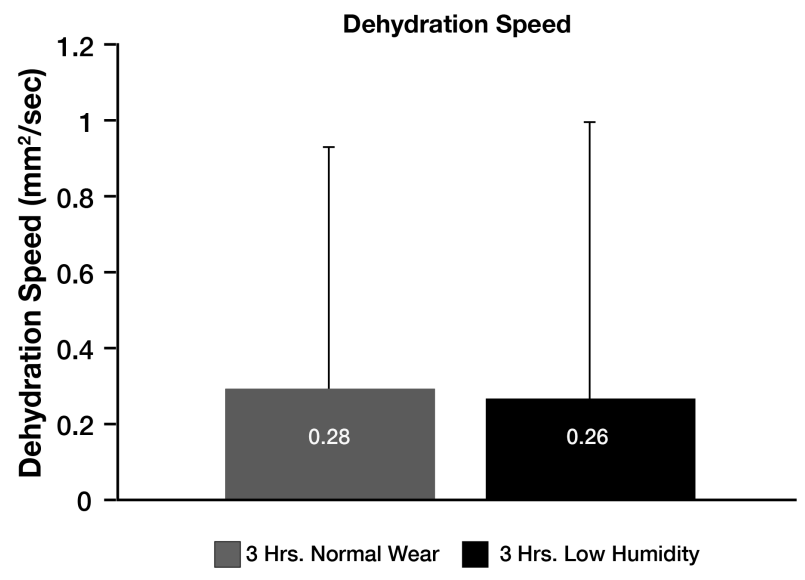

Figure 3. Mean \pm SD dehydration speed for habitual delefilcon A wearers after 3 hours of normal wear and 3 hours of exposure to $20 \%$ relative humidity. 


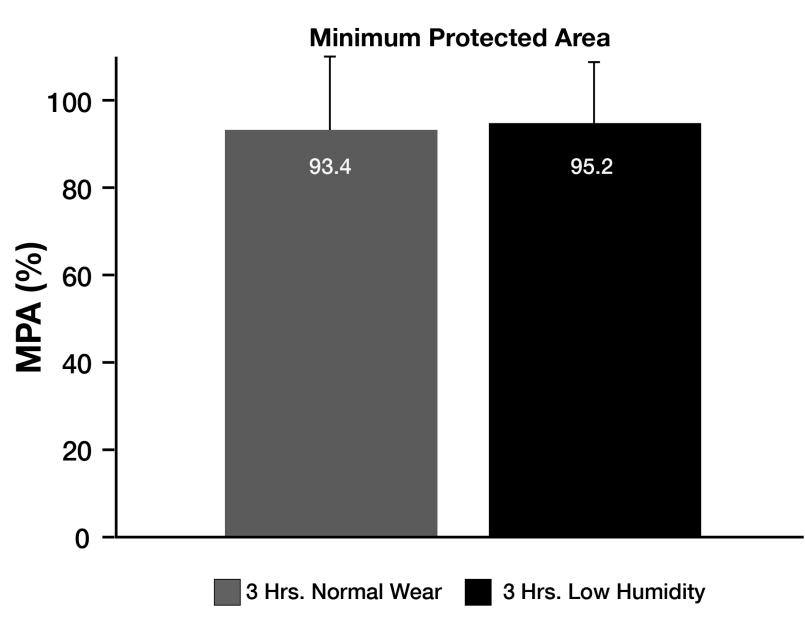

Figure 4. Mean \pm SD percent minimum protected area (MPA) for habitual delefilcon A wearers after 3 hours of normal wear and 3 hours of exposure to $20 \%$ relative humidity.

additional 3 hours of intensive computer work in a lowhumidity environment (i.e., AEC), indicating that good on-eye wettability was maintained under low-humidity conditions while performing computer activities.

\section{TFK Reproducibility in Study 1 vs. Study 2}

\section{Normal environment: 3 hours lens wear}

After 3 hours of conventional wear, the mean [95\% $\mathrm{CI}]$ differences between the two studies were $-0.9[-3.2$, $+1.4]$ seconds for NIBUT, $0.00[-0.22,+0.22] \mathrm{mm}^{2} / \mathrm{s}$ for DS, and $2.0[-3.5,+7.4] \%$ for MPA (Figure 5). Hence, TFK characteristics after the first 3 hours of wear were similar for the two studies, as demonstrated by the small mean differences recorded and two-sided 95\% CI for all the parameters.

\section{Adverse environment: 3 hours in 20\% RH}

After an additional 3 hours of wear in reduced $\mathrm{RH}$ of $20 \%$, the mean [ $95 \% \mathrm{CI}]$ differences between the two studies were $-1.3[-4.5,+1.9]$ seconds for NIBUT, $-0.03[-0.28,+0.22] \mathrm{mm}^{2} / \mathrm{s}$ for DS and $-0.3[-4.9,+4.3] \%$ for MPA (Figure 6). Therefore, TFK characteristics after 3 hours of wear of this lens material while carrying out computer activities in a low-humidity environment (AEC) remained similar for the two studies as demonstrated by the small mean differences recorded and two-sided 95\% CI for all parameters.

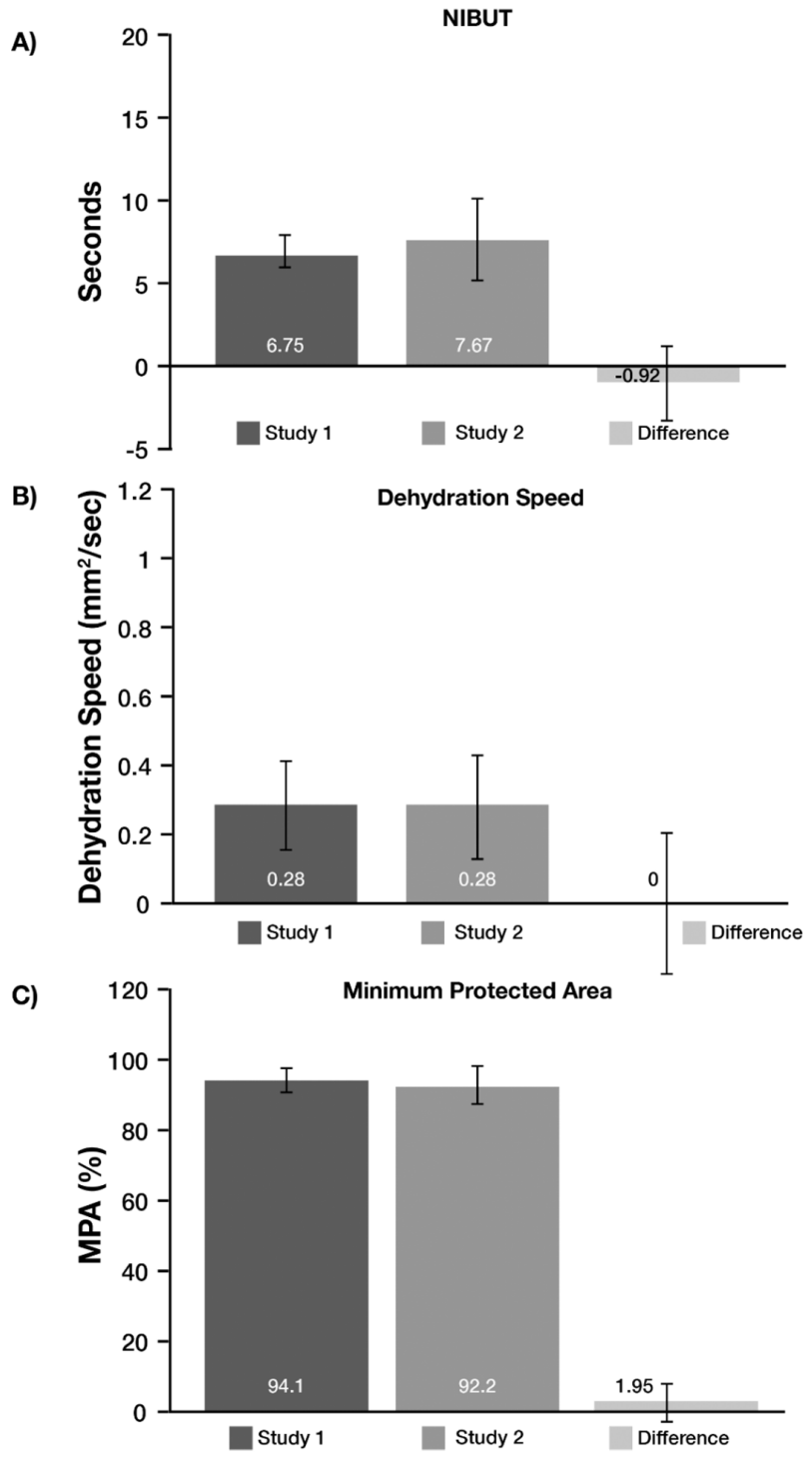

Figure 5. Mean $[95 \% \mathrm{CI}]$ (A) non-invasive breakup time (NIBUT), (B) dehydration speed, and (C) minimum protected area in a normal environment for Study 1 and Study 2 and the difference between Studies 1 and 2

\section{DISCUSSION}

The main objective of the analysis was to evaluate the reproducibility of TFK measurements during the entire interblink period in habitual wearers of delefilcon A daily disposable contact lenses under different environmental conditions. The data obtained showed that these measurements were highly reproducible, with 


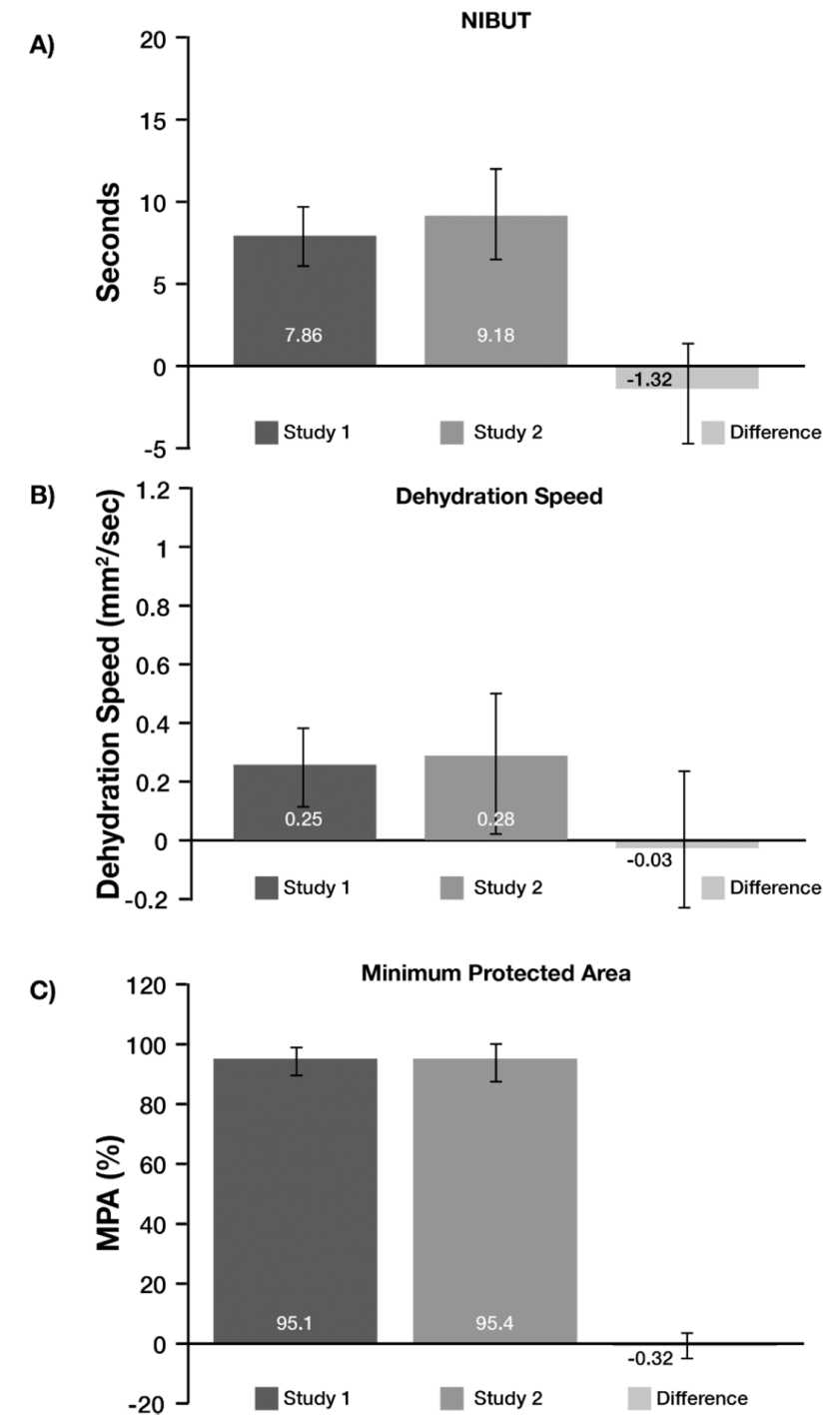

Figure 6. Mean [95\% CI] (A) non-invasive break-up time (NIBUT), (B) dehydration speed, and (C) minimum protected area (MPA) at $20 \%$ relative humidity for Study 1 and Study 2 and the difference between Studies 1 and 2.

mean $[95 \% \mathrm{CI}]$ differences between the two studies of $-0.9[-3.2,+1.4]$ seconds for NIBUT, $0.00[-0.22$, $+0.22] \mathrm{mm}^{2} / \mathrm{s}$ for DS, and $2.0[-3.5,+7.4] \%$ for MPA. Moreover, the Tearscope video recording method was reliable for quantifying pre-contact lens TFK and could therefore be used to assess contact lens on-eye wettability in clinical studies. These data, therefore, meet the objective of this study and support the hypothesis tested.
Analysis of TFK parameters in contact lens wearers allows the quantification of tear film dynamics over the entire interblink period. ${ }^{3}$ These TFK parameters included NIBUT coverage of the contact lens surface by the tear film; lens surface coverage by tears at the time of the blink (MPA), when the eyelid interacts with the contact lens; and the speed at which the tear film destabilizes once the blink has occurred (DS). This is indicative of surface resistance to dehydration in vivo and analogous to measuring the receding angle of the contact lens surface in vivo. The studies utilized this methodology to evaluate contact lens wearers under normal environmental conditions and following prolonged and intensive computer use under the controlled low-humidity conditions encountered in air-conditioned offices. ${ }^{9,10}$

The large body of data collected approximately one year apart in two studies of similar protocols enables conclusions as to on-eye wettability of delefilcon A contact lenses. The overall data showed that on-eye wettability, as determined by detailed TFK analysis, was normal after the first 3 hours of wear and remained normal after an additional 3 hours of wear under lowhumidity conditions while carrying out controlled, intensive computer tasks. These findings support the recommendations that delefilcon A contact lenses be prescribed for subjects involved with intensive device use and subjects exposed to adverse hygrometric environments for sustained periods. Additionally, this analysis also showed that delefilcon A TFK were normal and consistent across studies, indicating that good on-eye wettability was a constant for the material, confirming results of previous studies involving delefilcon A. ${ }^{11-14}$

To our knowledge, this report is the first to determine that measurements of pre-contact lens tear film characteristics were reproducible. First, these characteristics had been identified in the TFOS DEWS

II Tear Film workshop report as essential parameters to quantify to judge the validity of a measurement method. Second, this report comparing two separate clinical studies established that the measurement of pre-contact lens TFK using the Tearscope is reproducible and, therefore, valid and of clinical application. Third, the measurements obtained to determine the standard deviation of the difference for this methodology 
provides the information required to calculate sample sizes for future studies using this same technique. Indeed, the comparison of NIBUT, DS, and MPA obtained in the two separate studies showed their similarity, confirming the reliability of this technique.

\section{CONCLUSION}

In conclusion, the TFK measurements produced repeatable results in two clinical studies characterizing contact lens on-eye wettability under normal and low-humidity conditions. The results reported enable precise sample size determination in future studies using the Tearscope method to measure pre-contact lens tear film stability. Delefilcon A contact lenses performed consistently in two individual clinical studies carried out approximately one year apart. Delefilcon A TFK was unchanged under challenging indoor environmental conditions while performing intensive computer visual tasks. Delefilcon A contact lenses are suitable for contact lens wearers involved in intensive usage of computers and digital devices.

\section{REFERENCES}

1. Sweeney DF, Millar TJ, Raju SR. Tear film stability: a review. Exp Eye Res 2013;117:28-38.

2. Craig JP, Willcox MDP, Argueso P, et al. The TFOS International Workshop on Contact Lens Discomfort: report of the contact lens interactions with the tear film subcommittee. Invest Ophthalmol Vis Sci 2013;54:123-156.

3. Guillon M, Dumbleton KA, Theodoratos P, et al. Association between contact lens discomfort and pre-lens tear film kinetics. Optom Vis Sci 2016;93:881-891.

4. Willcox MDP, Argueso P, Georgiev GA, et al. TFOS DEWS II Tear Film Report. Ocul Surface 2017;15:369-406.
5. Bartlett JW, Frost C. Reliability, repeatability and reproducibility: analysis of measurement erros in continuous variables. Ultrasound Obstet Gynecol 2008: 31:466-475.

6. Thekveli S, Bauman E. Comparison of the surface morphology of daily disposable silicone hydrogel contact lenses via atomic force microscopy. Cont Lens Anterior Eye Suppl. 2013; 36:e30 (abstract).

7. Thekveli S, Qiu Y, Kapoor Y, Liang W, Pruitt J. Structure-property relationship of delefilcon A lenses. Cont Lens Anterior Eye Suppl. 2012; $35:$ : 14 (abstract).

8. Rantamaki AH, Holopainen JM. The effect of phospholipids on tear film lipid layer surface activity. Invest Ophthalmol Vis Sci. 2017;58:149-154.

9. Kojima T, Ibrahim OM, Wakamatsu T, et al. The impact of contact lens wear and visual display terminal work on ocular surface and tear functions in office workers. Am J Ophthalmol 2011;152:933-940.

10. Maruyama K, Yokoi N, Takamata A, Kinoshita S. Effect of environmental conditions on tear dynamics in soft contact lens wearers. Invest Ophthalmol Vis Sci 2004;45:2563-2568.

11. Wolffsohn JS, Mroczko S, Bilkhu P, et al. Crossover evaluation of silicone hydrogel daily disposable contact lenses. Optom Vis Sci 2015;92:1063-1068. .

12. Varikooty J, Schulze MM, Dumbleton K, et al. Clinical performance of three silicone hydrogel daily disposable lenses. Optom Vis Sci 2015;93:301-311

13. Szczesna-Iskander DH. Comparison of tear film surface quality measured in vivo on water gradient silicone hydrogel and hydrogel contact lenses. Eye Contact Lens 2014;40: 23-27.

14. Michaud L, Forcier P. Comparing two different daily disposable lenses for improving discomfort related to contact lens wear. Cont Lens Anterior Eye 2016;239:203-209.

J Cont Lens Res Sci Vol 5(1):e11-e18; September 30, 2021

This article is distributed under the terms of the Creative Commons Attribution-Non Commercial 4.0 International License. (CGuillon et al. 